\title{
ON ADDITION THEOREMS RELATED TO ELLIPTIC INTEGRALS
}

\author{
MALKHAZ BAKURADZE AND VLADIMIR VERSHININ
}

\begin{abstract}
We give the formulæfor the components of the Buchstaber formal group law and its exponent over $\mathbb{Q}\left[p_{1}, p_{2}, p_{3}, p_{4}\right]$. This leads to an addition theorem for the general elliptic integral $\int_{0}^{x} d t / R(t)$, for $R(t)=\sqrt{1+p_{1} t+p_{2} t^{2}+p_{3} t^{3}+p_{4} t^{4}}$. The motivation comes from Euler's addition theorem for elliptic integrals of the first kind.
\end{abstract}

\section{Introduction AND STATEMENTS}

The Jacobi elliptic sine is the elliptic version of the circular sine. In traditional notation, $\operatorname{sn}(u)$ is the inversion of the elliptic integral of the first kind

$$
\operatorname{sn}(u)=x, u=\int_{0}^{x} \frac{d t}{\sqrt{\left(1-t^{2}\right)\left(1-k^{2} t^{2}\right)}},
$$

where $k$ is some parameter called modulus.

Addition theorems offer a means of determining the value of the function for the sum of two quantities as arguments, when the values of the function for each argument is known.

The function $f(u)=\operatorname{sn}(u)$ satisfies Euler's addition theorem

$$
f(u+v)=\frac{f(u) R(f(v))+f(v) R(f(v))}{1-k^{2} f(u)^{2} f(v)^{2}}, \quad \text { where } R(u)=\sqrt{\left(1-u^{2}\right)\left(1-k^{2} u^{2}\right)} .
$$

Let us rewrite (1.1) in the form of the addition law due to Cayley [16], (see also [9], (1.4)).

$$
f(u+v)=\frac{f^{2}(u)-f^{2}(v)}{f(u) f^{\prime}(v)-f(v) f^{\prime}(u)}, \quad f(0)=0, \quad f^{\prime}(0)=1 .
$$

The further solutions of (1.2) are as follows.

The exponent series of the Ochanine elliptic genus $f=\exp \left(\phi_{O c}\right)$ over $\mathbb{Q}[\delta, \epsilon]$ is defined as the inversion of the elliptic integral

$$
\int_{0}^{x} \frac{d t}{\sqrt{1+\delta t^{2}+\epsilon t^{4}}}
$$

Let $f_{0}(u)=1 / \Phi(u)$, where $\Phi(u)=\Phi(u, \alpha)$ is the simplest Baker-Akhiezer function [11]. One has the addition formula [4]

$$
f_{0}(u+v)=\frac{f_{0}^{2}(u)\left(\frac{f_{0}(v)}{-f_{0}(-v)}\right)-f_{0}^{2}(v)\left(\frac{f_{0}(u)}{-f_{0}(-u)}\right)}{f_{0}(u) f_{0}^{\prime}(v)-f_{0}(v) f_{0}^{\prime}(u)} .
$$

For other interesting examples see [4], [7], [8].

2010 Mathematics Subject Classification. 33E05, 55N22.

Key words and phrases. Addition theorem, Complex elliptic genus, Formal group law.

The first author was supported by CNRS PICS N 7736, and by Shota Rustaveli NSF grant 217-614.

CNRS PICS N 7736. 
Consider the universal Buchstaber formal group law, the universal example of the formal group law of the form

$$
F(x, y)=\frac{x^{2} A(y)-y^{2} A(x)}{x B(y)-y B(x)}
$$

also specializing to the Euler formal group law (1.1) for $A(x)=1$ and $B(x)=R(x)$.

It is known from [4] that the exponent series of (1.5) gives the universal example of $f(u)$ such that $f(0)=0, f^{\prime}(0)=1$ and such that $f$ has an addition theorem of the form

$$
f(u+v)=\frac{f(u)^{2} \xi_{1}(v)-f(v)^{2} \xi_{1}(u)}{f(u) \xi_{2}(v)-f(v) \xi_{2}(u)},
$$

for some series $\xi_{1}(u)=A(f(u))$ and $\xi_{2}(u)=B(f(u))=f^{\prime}(u)$ such that $\xi_{1}(0)=\xi_{2}(0)=1$.

By $[10] f$ is defined over the polynomial ring $\mathbb{Q}\left[p_{1}, p_{2}, p_{3}, p_{4}\right]$ on four variables of degrees 2,4 , 6 and 8.

Our main result is Theorem 1.1. It provides new explicit formulæ(1.8) and (1.11) for the series $B$ and $A$ in (1.5) after tensoring the coefficient ring of $F$ with rationals, i.e., over $\Lambda \otimes \mathbb{Q}=$ $\mathbb{Q}\left[p_{1}, p_{2}, p_{3}, p_{4}\right]$. It also provides the differential equation (1.10) with the general solution $\mathcal{B}$.

Let us define the following formal power series over the polynomial ring $\mathbb{Q}\left[p_{1}, p_{2}, p_{3}, p_{4}\right]$, where $p_{1}, \cdots, p_{4}$ are variables of degrees $2,4,6$ and 8 respectively.

$$
\begin{aligned}
\mathcal{R}(x) & :=\sqrt{1+p_{1} x+p_{2} x^{2}+p_{3} x^{3}+p_{4} x^{4}} ; \\
\mathcal{B}(x) & :=\frac{\mathcal{R}(\mu(x))}{\mu^{\prime}(x)} \quad \text { i.e., } \mathcal{R}(x)=\frac{\mathcal{B}(\nu(x))}{\nu^{\prime}(x)}, \\
\text { where } \quad \mu(x) & :=\frac{x}{\mathcal{B}(x)}, \quad \nu(x):=\mu^{-1}(x)
\end{aligned}
$$

Thus $\mathcal{B}(x), \mathcal{B}(0)=1$ is a general solution of the differential equation

$$
\begin{gathered}
\mathcal{B}(x)^{2}\left(\mathcal{B}(x)-x \mathcal{B}^{\prime}(x)\right)^{2}=\mathcal{B}(x)^{4}+p_{1} x \mathcal{B}(x)^{3}+p_{2} x^{2} \mathcal{B}(x)^{2}+p_{3} x^{3} \mathcal{B}(x)+p_{4} x^{4} . \\
\mathcal{A}(x):=\mathcal{B}^{2}(x)-\frac{1}{2} x \mathcal{B}(x) \mathcal{B}^{\prime}(x)+\frac{1}{4} p_{1} x \mathcal{B}(x)-\left(\frac{1}{16} p_{1}^{2}-\frac{1}{4} p_{2}\right) x^{2} .
\end{gathered}
$$

Note that all series above are defined in terms of $\mathcal{R}(x)$.

Theorem 1.1. In notations (1.5) - (1.9) and (1.11) one has over the polynomial ring $\mathbb{Q}\left[p_{1}, p_{2}, p_{3}, p_{4}\right]$

$$
A(x)=\mathcal{A}(x), \quad B(x)=\mathcal{B}(x), \text { and } \log _{F}(x)=\int_{0}^{x} \frac{d t}{\mathcal{B}(t)} .
$$

Theorem 1.1 leads to the following

Corollary 1.2. Let $f(u)$ be a formal power series over a $\mathbb{Q}$-algebra such that $f(0)=0, f^{\prime}(0)=1$. Then $f$ has an addition theorem of the form (1.6) if and only if it satisfies one of the following properties

i) $f$ is the inversion of $u=\int_{0}^{x} \frac{d t}{\mathcal{B}(t)}$, or equivalently $f$ is a solution of $f^{\prime}=\mathcal{B}(f)$.

ii) The series

satisfies the differential equation

$$
h(x):=\frac{f^{\prime}(x)}{f(x)}
$$

$$
\left(h^{\prime}(x)\right)^{2}=S(h(x))
$$


where $S$ is the generic monic polynomial of degree 4 :

$$
S(t)=p_{4}+p_{3} t+p_{2} t^{2}+p_{1} t^{3}+t^{4} .
$$

Taking into account the remarks after (1.6) one has the following

Corollary 1.3. The following series $\xi_{1}$ and $\xi_{2}$ fit into (1.6)

$$
\begin{aligned}
& \xi_{1}(u)=\mathcal{A}(f(u))=f^{\prime 2}(u)-\frac{1}{2}\left(f^{\prime \prime}(u)+f^{\prime \prime}(0) f^{\prime}(u)\right) f(u)+\frac{1}{2}\left(f^{\prime \prime 2}(0)-f^{\prime \prime \prime}(0)\right) f^{2}(u) ; \\
& \xi_{2}(u)=\mathcal{B}(f(u))=f^{\prime}(u) .
\end{aligned}
$$

In [8] the proof of Theorem E.5.4 derives the formulæwhich agree with (1.12), (1.13). In particular, the coefficient $b_{1}$ of $x$ in $B(x)$ does not affect on (1.5), and therefore can be chosen arbitrarily. Similarly, the series $\xi_{1}(u)=A(f(u))$ and $\xi_{2}(u)=B(f(u))$ in (1.6) are defined up to summands $k_{1} f(u)^{2}$ and $k_{2} f(u)$, for any constants $k_{1}$ and $k_{2}$ respectively. We derive (1.11) and (1.12) using (2.4) of Lemma 2.2.

The following is the addition theorem for general elliptic integral and its inversion $S N(u)$, which specializes to elliptic sine. In particular it is defined by

$$
S N(u)=x, u=\int_{0}^{x} \frac{d t}{\mathcal{R}(t)},
$$

where $\mathcal{R}$ is as in (1.7). The function $S N$ is the exponent series of a genus $\psi$ introduced in [13].

Theorem 1.4. One has the addition formula

$$
\int_{0}^{x} \frac{d t}{\mathcal{R}(t)}+\int_{0}^{y} \frac{d t}{\mathcal{R}(t)}=\int_{0}^{G(x, y)} \frac{d t}{\mathcal{R}(t)}
$$

where

$$
G(x, y)=\mu\left(P_{1}+\sigma P_{1}+\frac{1}{2} \nu(x) \nu(y) \frac{P_{2}-\sigma P_{2}}{P_{3}-\sigma P_{3}}\right),
$$

here $\sigma$ is the transposition of $x$ and $y$ and the series $P_{i}=P_{i}(x, y)$ are determined by

$$
\begin{aligned}
& P_{1}=\nu(x) \mathcal{R}(y) \nu^{\prime}(y), \\
& P_{2}=-\nu(x) \nu^{\prime}(y)\left(\mathcal{R}(y)\left(\mathcal{R}^{\prime}(y)-\mathcal{R}^{\prime}(0)\right)-\nu(x) \mathcal{R}^{2}(y) \nu^{\prime \prime}(y)\right), \\
& P_{3}=\nu(x) \mathcal{R}(y) \nu^{\prime}(y) .
\end{aligned}
$$

Corollary 1.5. Let $S N(x)$ be the inversion of $\int_{0}^{x} \frac{d t}{\mathcal{R}(t)}$, then

$$
S N(x+y)=G(S N(x), S N(y)) .
$$

To prove Theorem 1.4 we first reduce the problem of explicit addition theorem to KricheverHöhn genus [10], which is defined over $\mathbb{Q}\left[p_{1}, p_{2}, p_{3}, p_{4}\right]$ by $(2.2)$. For this we use the explicit strict isomorphism of Lemma 2.1. From [2] we know that the universal Buchstaber formal group law $F_{B}$ (1.5) can be alternatively defined by the Nadiradze genus $\phi_{N}$ (2.3). In Theorem 2.3 we prove that the Krichever-Höhn genus is identical to the Nadiradze genus $\phi_{N}$ after rationalization of its values ring $\Lambda$, i.e., over $\Lambda \otimes \mathbb{Q}=\mathbb{Q}\left[p_{1}, p_{2}, p_{3}, p_{4}\right]$. This reduces the task to the universal Buchstaber formal group law $F_{B}$ and we apply our explicit formulæfor the components of $F_{B}$ obtained in Theorem 1.1 . 


\section{PRELIMINARIES}

It is convenient to give proofs of our results in terms of formal group laws. We give here the necessary definitions and facts. We refer the reader to [6] as the detailed survey in the subject.

A formal group law over a commutative ring with unit $R$ is a formal power series $F(x, y)$ in $R[[x, y]]$ satisfying

(i) $F(x, 0)=F(0, x)=x$,

(ii) $F(x, y)=F(y, x)$,

(iii) $F(x, F(y, z))=F(F(x, y), z)$.

Let $F$ and $G$ be formal group laws. A homomorphism from $F$ to $G$ is a power series $h(x) \in R[[x]]$ with constant term 0 such that

$$
h(F(x, y))=G(h(x), h(y)) .
$$

It is an isomorphism if $h^{\prime}(0)$ (the coefficient at $x$ ) is a unit in $R$, and a strict isomorphism if the coefficient at $x$ is 1 .

If $F$ is a formal group law over a commutative $\mathbb{Q}$-algebra $R$, then it is strictly isomorphic to the additive formal group law $x+y$. In other words, there is a strict isomorphism $l(x)$ from $F$ to the additive formal group law. The series $l(x)$ is called the logarithm of $F$, so we have $F(x, y)=l^{-1}(l(x)+l(y))$. The inverse to logarithm is called the exponential of $F$.

The logarithm $\log _{F}(x):=l(x) \in R \otimes \mathbb{Q}[[x]]$ of a formal group law $F$ is given by

$$
\log (x)=\int_{0}^{x} \frac{d t}{\omega(t)}, \quad \omega(x)=\frac{\partial F(x, y)}{\partial y}(x, 0) .
$$

We will often use the following consequence of definitions above: If $h(x)$ is a strict isomorphism from the formal group law $F$ to $G$, then

$$
\log _{F}=\log _{G}(h), \quad \text { i.e., } \log _{G}=\log _{F}\left(h^{-1}\right) .
$$

There is a ring $\mathbf{L}$, called the universal Lazard ring, and a universal formal group law $F(x, y)=$ $\sum a_{i j} x^{i} y^{j}$ defined over $\mathbf{L}$. This means that for any formal group law $F^{\prime}$ over any commutative ring with unit $R$ there is a unique ring homomorphism $r: \mathbf{L} \rightarrow R$ such that $F^{\prime}(x, y)=\sum r\left(a_{i j}\right) x^{i} y^{j}$.

The formal group law of geometric cobordism was introduced in [14]. Following Quillen we will identify it with the universal Lazard formal group law as it is proved in [15] that the coefficient ring of complex cobordism $\mathbf{M U}^{*}=\mathbb{Z}\left[x_{1}, x_{2}, \ldots\right],\left|x_{i}\right|=2 i$ is naturally isomorphic as a graded ring to the universal Lazard ring.

The coefficients of the formal group law of geometric cobordisms $F_{U}$ and its logarithm may be described geometrically by the following results.

Theorem (Buchstaber [5]).

$$
F_{U}(u, v)=\sum_{i, j \geq 0}\left[H_{i j}\right] u^{i} v^{j}\left(\sum_{r \geq 0}\left[\mathbf{C P}_{r}\right] u^{r}\right)\left(\sum_{s \geq 0}\left[\mathbf{C P}_{s}\right] v^{s}\right)
$$

where $\mathbf{C P}_{r}$ are the complex projective spaces, $H_{i j}(0 \leq i \leq j)$ are Milnor hypersurfaces and $H_{j i}=H_{i j}$.

Theorem (Mishchenko, see [14]). The logarithm of the formal group law of geometric cobordisms is given by the series

$$
\log (u)=u+\sum_{k \geq 1} \frac{\left[\mathbf{C P}_{k}\right]}{k+1} u^{k+1} \in \mathbf{M U}^{*} \otimes \mathbb{Q}[[u]]
$$

The addition formula (1.1) corresponds to Euler's addition formula for the elliptic integrals of the first kind

$$
\int_{0}^{x} \frac{d t}{\sqrt{\left(1-t^{2}\right)\left(1-k^{2} t^{2}\right)}}+\int_{0}^{y} \frac{d t}{\sqrt{\left(1-t^{2}\right)\left(1-k^{2} t^{2}\right)}}=\int_{0}^{T(x, y)} \frac{d t}{\sqrt{\left(1-t^{2}\right)\left(1-k^{2} t^{2}\right)}}
$$


where

$$
T(x . y)=\frac{x \sqrt{\left(1-y^{2}\right)\left(1-k^{2} y^{2}\right)}+y \sqrt{\left(1-x^{2}\right)\left(1-k^{2} x^{2}\right)}}{1-k^{2} x^{2} y^{2}}
$$

is the Euler formal group law. In terms of the logarithm of the formal group law this means

The following two ideas come naturally in mind.

First, one can replace $\sqrt{\left(1-x^{2}\right)\left(1-k^{2} x^{2}\right)}$ by more general expression (1.7) and consider the corresponding formal group law $G(x, y)$ with logarithm

$$
\log _{G}=\int_{0}^{x} \frac{d t}{\mathcal{R}(t)}
$$

specializing to the elliptic integral of the first kind. The corresponding formal group law was recently studied in [13].

Second, one can consider the universal Buchstaber formal group law, the universal example of the formal group law of the form (1.5).

Motivated by string theory, the general complex elliptic genus (also called Krichever-Höhn genus) has been defined: in [11] Krichever wrote down its characteristic power series $Q(x)$ using Baker-Akhiezer function. In [10], Höhn defined four variable elliptic genus $\phi_{K R}$ determined by the following property: if one denotes by $f=f_{K H}$ the exponent of the corresponding formal group $F_{K H}$, then the series

satisfies the differential equation

$$
h(x):=\frac{f^{\prime}(x)}{f(x)}
$$

$$
\left(h^{\prime}(x)\right)^{2}=S(h(x)),
$$

where $S$ is the generic monic polynomial of degree 4 ,

$$
S(t)=p_{4}+p_{3} t+p_{2} t^{2}+p_{1} t^{3}+t^{4} .
$$

He also showed that $\phi_{K R}$ takes values in the ring $\mathbb{Q}\left[p_{1}, p_{2}, p_{3}, p_{4}\right]$ and that it agrees with Krichevers denition.

We use the observation that these two formal groups $G$ and $F=F_{K H}$ are connected by an explicit isomorphism. In particular

Lemma 2.1. The series $\frac{x}{\mathcal{B}(x)}$ is the strict isomorphism from $F_{K H}$ to $G$.

For the proof see [1] or [3]. Note that on page 13 in [1] the value $\psi(\mathbb{C} P)$ should read as

$$
\frac{35}{128} q_{1}^{4}-\frac{15}{16} q_{1}^{2} q_{2}+\frac{3}{8} q_{2}^{2}+\frac{3}{4} q_{1} q_{3}-\frac{1}{2} q_{4}
$$

We shall need some results of [1], [2] and we collect them together in the following.

Recall here how the universal Nadiradze formal group $F_{N}$ is constructed [2]. Let $F_{U}$ be the universal formal group law over MU*. Define the series

$$
\sum A_{i j} x^{i} y^{j}=F_{U}(x, y)(x \omega(y)-y \omega(x)) .
$$

Now kill all $A_{i, j}$, with $i, j \geq 3$. Then the Nadiradze formal group law is classified by the quotient map

$$
\phi=\phi_{N}: \mathbf{M U}^{*} \rightarrow \mathbf{M U}^{*} /\left(A_{i, j}, i, j \geq 3\right):=\Lambda .
$$

In other words $F_{N}$ is the universal formal group law whose invariant differential $\omega(x) \in \Lambda[[x]]$ is

$$
\frac{\partial F(x, y)}{\partial y}(x, 0)=1 / \phi(\mathbf{C P}(x))=1 /\left(1+\sum \phi\left[\mathbf{C P}_{i}\right] x^{i}\right) .
$$


Let $F_{B}$ be the universal Buchstaber formal group law, the universal example among the formal group laws of the form (1.5).

Lemma 2.2. [2] i) The universal Nadiradze formal group law $F_{N}$ over $\Lambda$ is identical to the universal Buchstaber formal group law $F_{B}$, i.e., $F_{B}$ is the formal groups of the form (1.5), where

$$
B(x)=1 / \phi(\mathbf{C P}(x)) .
$$

ii) The series $A(x) \in \Lambda[[x]]$ can be defined by

$$
A(x)=-x^{2} B(x) \beta(x)-b_{1} x B(x)+B^{2}(x)-b_{2} x^{2},
$$

where

$$
\beta(x)=\frac{B^{\prime}(x)-b_{1}}{2 x} \in \Lambda[[x]]
$$

and $b_{1}, b_{2}$ are the coefficients of $B(x)=1+\sum_{i \geq 1} b_{i} x^{i}$.

Note that for the formula (2.4) we have to use the series $\sum A_{i j} x^{i} y^{j}$ in the proof of Proposition 2 in [2] and rewrite $A(x)=\sum A_{i 2} x^{i}$ and $B(x)=\omega(x)$.

One has also the following

Theorem 2.3. Let $\Lambda$ be the ring of coefficients of the universal Nadiradze formal group $F_{N}$. Then $F_{N}$ over $\Lambda \otimes \mathbb{Q}=\mathbb{Q}\left[p_{1}, p_{2}, p_{3}, p_{4}\right]$ is identical to the Krichever-Höhn formal group law $F_{K H}$.

\section{Proofs}

Proof of Theorem 2.3. By [10] the coefficient ring $\Lambda$ of the universal Buchstaber formal group law tensored by rationals is

$$
\Lambda \otimes \mathbb{Q}=\mathbb{Q}\left[p_{1}, p_{2}, p_{3}, p_{4}\right],
$$

therefore it suffices to consider the formal group laws over $\mathbb{Q}\left[p_{1}, p_{2}, p_{3}, p_{4}\right]$

Let $l=\log _{G}$ be the logarithm of the formal group law $G$ in Lemma 2.1 and let $j(x)=1 / l^{\prime}$. Lemma 2.1 says that for a formal group law $F$, the condition of Höhn (2.2) is satisfied if and only if $j(x)^{2}$ is a polynomial of degree 4 with constant term 1 .

Let $\phi$ be the classifying map of a Buchstaber formal group law and let

$$
C(x)=\sum C_{i} x^{i}=\phi(\mathbf{C P}(x))=1+\sum_{i \geq 1} \phi\left[\mathbf{C P}_{i}\right] x^{i} .
$$

By Lemma 2.2 i) necessarily we have

$$
B(x)=1 / C(x),
$$

so that we obtain

$$
A(x, y):=F(x, y)(x / C(y)-y / C(x))=x^{2} A(y)-y^{2} A(x) .
$$

It follows that

$$
\frac{\partial^{3} A}{\partial y^{3}}(x, 0)=c x^{2}
$$

for some constant $c$.

Taking into account that $F(x, y)=f(g(x)+g(y))$, that $g(0)=0, g^{\prime}(0)=1$, that $f(g(x))=x$, and that $f^{\prime}(g(x))=1 / g^{\prime}(x)=1 / C(x)$ one obtains

$$
\begin{aligned}
& \frac{\partial^{3} A}{\partial y^{3}}(x, 0)=-\frac{x C^{\prime \prime}(x)}{C(x)^{4}}+\frac{3 x C^{\prime}(x)^{2}}{C(x)^{5}}+\frac{3 C^{\prime}(x)}{C(x)^{4}}-\frac{3 C_{1}}{C(x)^{2}}+\frac{\left(3 C_{1}^{2}-4 C_{2}\right) x}{C(x)} \\
& +\left(-6 C_{1}^{3}+12 C_{1} C_{2}-4 C_{3}\right) x^{2}
\end{aligned}
$$

so that the series $C(x)$ satisfies the differential equation

$$
x C(x) C^{\prime \prime}(x)-3 x C^{\prime}(x)^{2}-3 C(x) C^{\prime}(x)+3 C_{1} C(x)^{3}-\left(3 C_{1}{ }^{2}-4 C_{2}\right) x C(x)^{4}+k x^{2} C(x)^{5}=0
$$


for some constant $k$.

Let us now substitute in this equation $\nu(x)$ in the place of $x$, where $\nu(x)$ is inversion of $x C(x)$, i. e. $\nu(x) C(\nu(x))=x$, so that

$$
\begin{aligned}
C(\nu(x)) & =\frac{x}{\nu(x)}, \\
C^{\prime}(\nu(x)) & =\nu^{\prime}(x)^{-1} \frac{\nu(x)-x \nu^{\prime}(x)}{\nu(x)^{2}}, \\
\nu(x) C(\nu(x)) C^{\prime \prime}(\nu(x)) & =-\nu^{\prime}(x)^{-3} \frac{x}{\nu(x)} \nu^{\prime \prime}(x)+2 \nu^{\prime}(x)^{-1} \frac{x}{\nu(x)} \frac{x \nu^{\prime}(x)-\nu(x)}{\nu(x)^{2}} .
\end{aligned}
$$

We then obtain that $\nu(x)$ satisfies the differential equation

$$
2 x \nu(x)^{2} \nu^{\prime \prime}(x)+\left(k x^{3}+\left(6 C_{1}^{2}-8 C_{2}\right) x^{2}-6 C_{1} x-1\right) x^{2} \nu^{\prime}(x)^{3}-2 x \nu(x) \nu^{\prime}(x)^{2}+6 \nu(x)^{2} \nu^{\prime}(x)=0 .
$$

Next let us consider

$$
j(x)=\frac{1}{l^{\prime}(x)}, \quad l=\log _{G}
$$

mentioned above.

By the strict isomorphism we have $l(x)=g(\nu(x))$. Hence

$$
\begin{aligned}
& f(l(x))=f(g(\nu(x)))=\nu(x), \\
& f^{\prime}(l(x)) l^{\prime}(x)=\nu^{\prime}(x),
\end{aligned}
$$

and taking into account

$$
\frac{f(l(x))}{f^{\prime}(l(x))}=1 / h\left((1 / h)^{-1}(x)\right)=x
$$

we have

$$
\begin{aligned}
j(x) & =\frac{\nu(x)}{x \nu^{\prime}(x)}, \\
\nu^{\prime}(x) & =\frac{\nu(x)}{x j(x)}, \\
\nu^{\prime \prime}(x) & =\frac{\nu(x)\left(1-j(x)-x j^{\prime}(x)\right)}{x^{2} j(x)^{2}} .
\end{aligned}
$$

Thus for the series $j$ we obtain the differential equation

$$
2 x j(x) j^{\prime}(x)=4 j(x)^{2}+k x^{3}+\left(6 C_{1}^{2}-8 C_{2}\right) x^{2}-6 C_{1} x-1 .
$$

Then further substituting $j(x)=\sqrt{p(x)}$ we obtain

$$
x p^{\prime}(x)=4 p(x)+k x^{3}+\left(6 C_{1}^{2}-8 C_{2}\right) x^{2}-6 C_{1} x-1
$$

and it is easy to see that the general solution of this equation is a fourth degree polynomial.

Conversely, by [4] $F_{K R}$ is identical to $F_{B}$ over $\mathbb{Q}\left[p_{1}, p_{2}, p_{3}, p_{4}\right]$ and by Lemma 2.2 i) $F_{B}$ is identical to $F_{N}$.

One can prove that $F_{K H}$ is a Buchstaber formal group law by Lemma 2.1 again. For $l=\log _{G}$ we have $l^{\prime}=\frac{1}{\mathcal{R}(x)}$ therefore we have for $\mu(x)=\frac{x}{\mathcal{B}(x)}, \nu=\mu^{-1}$ and $g:=\log _{F_{K H}}=l(\mu)$

$$
g^{\prime}=(l(\mu))^{\prime}=\frac{1}{\mathcal{R}(\mu))} \mu^{\prime}:=\frac{1}{\mathcal{B}} .
$$

Therefore differentiating the inverse function we get

$$
f^{\prime}=\mathcal{B}(f) \text {. }
$$

By Corollary $1.2 \mathrm{i}$ ) the corresponding formal group law is the Buchstaber formal group law over $\Lambda \otimes \mathbb{Q}=\mathbb{Q}\left[p_{1}, p_{2}, p_{3}, p_{4}\right]$ classified by the obvious inclusion $\Lambda \rightarrow \Lambda \otimes \mathbb{Q}$. 
Proof of Theorem 1.1. Let $l_{G}$ and $l_{F_{K H}}$ be the logarithms of the formal group laws $G$ and $F_{K H}$ respectively in Lemma 2.1. Then because of the strict isomorphism of Lemma $2.1 \mu(x)=\frac{x}{\mathcal{B}(x)}$ one has $l_{F_{K R}}(x)=l_{G}(\nu(x))$. By definition (2.1) of $l_{G}$ we have (1.8) and $l_{F_{K R}}(x)=\int_{0}^{x} \frac{d t}{\mathcal{B}(t)}$. But the latter coincides with the logarithm of the universal Buchstaber formal group law by Theorem 2.3 .

Now let us prove (1.11) for the series $A$ and $B$ over $\mathbb{Q}\left[p_{1}, p_{2}, p_{3}, p_{4}\right]$. By the note after Lemma 2.2 one has

$$
\begin{aligned}
& \mathcal{A}(x):=-x^{2} \mathcal{B}(x) \beta(x)-b_{1} x \mathcal{B}(x)+\mathcal{B}^{2}(x)-b_{2} x^{2}, \\
& \beta(x)=\frac{\mathcal{B}^{\prime}(x)-\mathcal{B}^{\prime}(0)}{2 x}=\frac{\mathcal{B}^{\prime}(x)-b_{1}}{2 x},
\end{aligned}
$$

or

$$
\mathcal{A}(x):=\mathcal{B}^{2}(x)-\frac{1}{2} x \mathcal{B}(x) \mathcal{B}^{\prime}(x)-\frac{1}{2} b_{1} x \mathcal{B}(x)-b_{2} x^{2} .
$$

Therefore it suffices to see

$$
b_{1}=-\frac{1}{2} p_{1} ; \quad b_{2}=\frac{1}{16} p_{1}^{2}-\frac{1}{4} p_{2} .
$$

By Lemma 2.2 i) and Theorem 2.3

$$
\begin{aligned}
\mathcal{B}(x)= & \phi(1 / \mathbf{C P}(x))=\frac{1}{\left(1+\phi\left[\mathbf{C P}_{1}\right] x+\phi\left[\mathbf{C P}_{2}\right] x^{2}+\cdots\right)}= \\
& 1-\phi\left[\mathbf{C P}_{1}\right] x+\phi\left(\left[\mathbf{C P}_{1}\right]^{2}-\left[\mathbf{C P}_{2}\right]\right) x^{2}+\cdots
\end{aligned}
$$

Then there is a formula of [3] for calculating the values of $\phi=\phi_{K H}$ on $\left[\mathbf{C P}_{i}\right]$, the generators of $\mathbf{M U}^{*} \otimes \mathbb{Q}$. In particular

$$
\begin{aligned}
& \phi\left(\left[\mathbf{C P}_{1}\right]=\frac{1}{2} p_{1} ;\right. \\
& \phi\left(\left[\mathbf{C P}_{1}\right]^{2}-\left[\mathbf{C P}_{2}\right]\right)=\frac{1}{4} p_{1}^{2}-\frac{3}{16} p_{1}^{2}-\frac{1}{4} p_{2}=\frac{1}{16} p_{1}^{2}-\frac{1}{4} p_{2} .
\end{aligned}
$$

This proves (1.11).

Proof of Corollary 1.2. i) follows directly from Theorem 1.1. For ii) apply Theorem 2.3. Here are some comments

Buchstaber's formal group law $F$ is of the form (3). Hence

$$
f(u+v)=F(f(u), f(v))=\frac{f(u)^{2} A(f(v))-f(v)^{2} A(f(u))}{f(u) B(f(v))-f(v) B(f(u))} .
$$

Thus $f$ has an addition theorem of the required form and

$$
\begin{aligned}
& \xi_{1}(v)=\mathcal{A}(f(u)), \\
& \xi_{2}(v)=\mathcal{B}(f(u)) .
\end{aligned}
$$

Let $f(u)$ be a series over any $\mathbb{Q}$-algebra, if it satisfies the addition theorem (1.6) and $\log =f^{-1}$, then the corresponding formal group law is as follows 
$F(u, v)=f(\log u+\log v)=\frac{f(\log u)^{2} \xi_{1}(\log v)-f(\log v)^{2} \xi_{1}(\log u)}{f(\log u) \xi_{2}(\log v)-f(\log v) \xi_{2}(\log u)}=\frac{u^{2} \xi_{1}(\log v)-v^{2} \xi_{1}(\log u)}{u \xi_{2}(\log v)-v \xi_{2}(\log u)}$.

By its form $F$ is a Buchstaber formal group law.

Proof of Corollary 1.3. (1.13) is just a differentiation of the inverse function in Theorem 1.1. (1.12): We have $\xi_{1}(u)=\mathcal{A}(f(u))$.

$$
\text { Let } f(x)=x+f_{1} x^{2}+f_{2} x^{3}+\cdots .
$$

Then by (1.13) we have $\mathcal{B}(f(x))=f^{\prime}(x)$, that is

$$
\begin{aligned}
& 1+b_{1}\left(x+f_{1} x^{2}+f_{2} x^{3}+\cdots\right)+b_{2}\left(x+f_{1} x^{2}+f_{2} x^{3}+\cdots\right)^{2}+\cdots= \\
& 1+2 f_{1} x+3 f_{2} x^{2}+\cdots
\end{aligned}
$$

hence

$$
\begin{aligned}
& b_{1}=2 f_{1}=f^{\prime \prime}(0) ; \\
& b_{2}=3 f_{2}-2 f_{1}^{2}=1 / 2 f^{\prime \prime \prime}(0)-1 / 2 f^{\prime \prime 2}(0) .
\end{aligned}
$$

Now taking into account $\mathcal{B}(f(u))=f^{\prime}(u)$ and $\mathcal{B}^{\prime}(f(u))=f^{\prime \prime}(u) / f^{\prime}(u)$ we get by $(1.11)$

$$
\begin{aligned}
\xi_{1}(u)=\mathcal{A}(f(u))= & \\
& \mathcal{B}(f(u))^{2}-\frac{1}{2} f(u) \mathcal{B}(f(u)) \mathcal{B}^{\prime}(f(u))-\frac{1}{2} b_{1} f(u) \mathcal{B}(f(u))-b_{2} f(u)^{2}= \\
& f^{\prime 2}(u)-\frac{1}{2} f(u) f^{\prime \prime}(u)-\frac{1}{2} b_{1} f(u) f^{\prime}(u)-b_{2} f(u)^{2}= \\
& f^{\prime 2}(u)-\frac{1}{2}\left(f^{\prime \prime}(u)+f^{\prime \prime}(0) f^{\prime}(u)\right) f(u)+\frac{1}{2}\left(f^{\prime \prime 2}(0)-f^{\prime \prime \prime}(0)\right) f^{2}(u) .
\end{aligned}
$$

3.1. Proof of Theorem 1.4. i) By Lemma 2.1 one has

$$
G(x, y)=\nu^{-1}\left[\frac{\nu(x)^{2} \mathcal{A}(\nu(y))-\nu(y)^{2} \mathcal{A}(\nu(x))}{\nu(x) \mathcal{B}(\nu(y))-\nu(y) \mathcal{B}(\nu(x))}\right]
$$

Then (1.8) and $\mu=\nu^{-1}$ imply

$$
\begin{aligned}
\mathcal{B}(\nu(x)) & =\frac{\mathcal{R}(\mu(\nu(x))}{\mu^{\prime}(\nu(x))}=\mathcal{R}(x) \nu^{\prime}(x), \\
\mathcal{B}^{\prime}(\nu(x)) & =\frac{\mathcal{B}(\nu(x))^{\prime}}{\nu^{\prime}(x)}=\frac{\left(\mathcal{R}(x) \nu^{\prime}(x)\right)^{\prime}}{\nu^{\prime}(x)}=\frac{\mathcal{R}^{\prime}(x) \nu^{\prime}(x)+\mathcal{R}(x) \nu^{\prime \prime}(x)}{\nu^{\prime}(x)}
\end{aligned}
$$

By (1.11) we have 


$$
\begin{aligned}
\mathcal{A}(\nu(x))= & \\
& \mathcal{R}^{2}(x)\left(\nu^{\prime}(x)\right)^{2}-\frac{1}{2} \nu(x) \mathcal{R}(x) \nu^{\prime}(x) \frac{\mathcal{R}^{\prime}(x) \nu^{\prime}(x)+\mathcal{R}(x) \nu^{\prime \prime}(x)}{\nu^{\prime}(x)}+ \\
& \frac{1}{4} p_{1} \nu(x) \mathcal{R}(x) \nu^{\prime}(x)-\left(\frac{1}{16} p_{1}^{2}-\frac{1}{4} p_{2}\right) \nu^{2}(x)= \\
& \mathcal{R}^{2}(x)\left(\nu^{\prime}(x)\right)^{2}-\frac{1}{2} \nu(x) \mathcal{R}(x) \mathcal{R}^{\prime}(x) \nu^{\prime}(x)+\frac{1}{4} p_{1} \nu(x) \mathcal{R}(x) \nu^{\prime}(x) \\
- & \frac{1}{2} \nu(x) \mathcal{R}^{2}(x) \nu^{\prime \prime}(x)-\left(\frac{1}{16} p_{1}^{2}-\frac{1}{4} p_{2}\right) \nu^{2}(x)= \\
& \mathcal{R}^{2}(x)\left(\nu^{\prime}(x)\right)^{2}-\frac{1}{2} \nu(x) \mathcal{R}(x) \nu^{\prime}(x)\left[\mathcal{R}^{\prime}(x)-\mathcal{R}^{\prime}(0)\right] \\
- & \frac{1}{2} \nu(x) \mathcal{R}^{2}(x) \nu^{\prime \prime}(x)-\left(\frac{1}{16} p_{1}^{2}-\frac{1}{4} p_{2}\right) \nu^{2}(x)
\end{aligned}
$$

as $\mathcal{R}^{\prime}(0)=\frac{1}{2} p_{1}$.

Now note that

$$
\frac{\mathcal{A}(\nu(y)) \nu^{2}(x)-\mathcal{A}(\nu(x)) \nu^{2}(y)}{\nu(x) \mathcal{R}(y) \nu^{\prime}(y)-\nu(y) \mathcal{R}(x) \nu^{\prime}(x)}=\nu(x) \mathcal{R}(y) \nu^{\prime}(y)+\nu(y) \mathcal{R}(x) \nu^{\prime}(x)+\frac{I+I I}{I I I}
$$

where

$$
\begin{gathered}
I=-\frac{1}{2} \nu^{2}(x) \nu(y) \nu^{\prime}(y) \mathcal{R}(y)\left[\mathcal{R}^{\prime}(y)-\mathcal{R}^{\prime}(0)\right]+\frac{1}{2} \nu^{2}(y) \nu(x) \nu^{\prime}(x) \mathcal{R}(x)\left[\mathcal{R}^{\prime}(x)-\mathcal{R}^{\prime}(0)\right] \\
I I=-\frac{1}{2} \nu^{2}(x) \nu(y) \mathcal{R}^{2}(y) \nu^{\prime \prime}(y)+\frac{1}{2} \nu^{2}(y) \nu(x) \mathcal{R}^{2}(x) \nu^{\prime \prime}(x) \\
I I I=\nu(x) \mathcal{R}(y) \nu^{\prime}(y)-\nu(y) \mathcal{R}(x) \nu^{\prime}(x) .
\end{gathered}
$$

Thus we get

$$
\nu(G(x, y))=\nu(x) \mathcal{R}(y) \nu^{\prime}(y)+\nu(y) \mathcal{R}(x) \nu^{\prime}(x)+\frac{1}{2} \nu(x) \nu(y) \frac{(P-\sigma P)}{\nu(x) \mathcal{R}(y) \nu^{\prime}(y)-\nu(y) \mathcal{R}(x) \nu^{\prime}(x)},
$$

where $\sigma \in S_{2}$ is the transposition of $x$ and $y$ and

$$
P=-\nu(x) \nu^{\prime}(y)\left(\mathcal{R}(y)\left(\mathcal{R}^{\prime}(y)-\mathcal{R}^{\prime}(0)\right)-\nu(x) \mathcal{R}^{2}(y) \nu^{\prime \prime}(y)\right) .
$$

This proves Theorem 1.4.

Finally, as usual, the formal group law $F=G$ gives the addition formula for its exponent $f=S N$

and Corollary 1.5 follows.

$$
f(u+v)=F(f(u), f(v))
$$

\section{REFERENCES}

[1] M. Bakuradze, On the Buchstaber formal group law and some related genera, Proc. Steklov Math. Inst., 286(2014), 7-21.

[2] M. Bakuradze, Formal group laws by Buchstaber, Krichever and Nadiradze coincide, Uspekhi Mat. Nauk, 68:3 (2013), 189-190.

[3] M. Bakuradze, Computing the Krichever genus, J. Homotopy Relat. Struct, 9, 1(2014), 85-93.

[4] V. M. Buchstaber, Functional equations associated with addition theorems for elliptic functions and two-valued groups, Uspekhi Mat. Nauk, 3, 273 (1990), 185-186.

[5] V. M. Buchstaber, Chern-Dold character in cobordisms. I, Mat. Sb. (N.S.) 83 (125) 1970, 575595. (Russian).

[6] V. M. Buchstaber, Complex cobordism and formal groups, Russian Math. Surv, 67:5, (2012), 891-950.

[7] V. M. Buchstaber, E.Yu. Bunkova, Krichever Formal groups, Funct. Anal. and appl. 45:2(2011), $23-44$. 
[8] V.M.Buchstaber, T.E.Panov, Toric Topology, Mathematical Surveys and Monographs, 204, Amer. Math. Soc., 2015.

[9] V. M. Buchstaber, A. V. Ustinov, Coefficient rings of formal group laws, Sb. Math., 206:11 (2015), 1524-1563.

[10] G. Höhn, Komplexe elliptische Geschlechter und $S^{1}$-äquivariante Kobordismustheorie, Bonn und Vallendar, August 1991. Available as arXiv:math/0405232

[11] I. Krichever, Generalized elliptic genera and Baker-Akhiezer functions, Math. Notes 47(1990), 132-142.

[12] S. Ochanine, Sur les genres multiplicatifs definis par des integrales elliptiques, Topology 26:2 (1987), $143-151$.

[13] S. Schreieder, Dualization invariance and a new complex elliptic genus, J. reine angew. Math.,2014.692 (2012): 77-108., arXiv:1109.5394v3 [math.AT], 2012.

[14] S. P. Novikov, The methods of algebraic topology from the viewpoint of cobordism theory, Math. USSR-Izv. 1:4 (1967), 827-913

[15] D. Quillen, On the formal group laws of unoriented and complex cobordism theory, Bull. Amer. Math. Soc. 75 (1969), 1293-1298.

[16] E.T. Whittaker and G.N. Watson, A course of modern analysis. An introduction to the general theory of infinite processes and of analytic functions; with an account of the principal transcendental functions, 4th ed., Cambridge Univ. Press, Cambridge 1927, vi+608 pp.

Faculty of exact and natural sciences, Iv. Javakhishvili Tbilisi State University, Georgia

E-mail address: malkhaz.bakuradze@tsu.ge

UNIVERSITY MOTPELLIER 2

E-mail address: vershini@uni-montp2.fr 